\title{
Salidroside inhibits the growth of human breast cancer in vitro and in vivo
}

\author{
GANG ZHAO, AIPING SHI, ZHIMIN FAN and YE DU \\ Department of Breast Surgery, The First Hospital, Jilin University, Changchun, Jilin 130021, P.R. China
}

Received December 10, 2014; Accepted January 26, 2015

DOI: $10.3892 /$ or.2015.3857

\begin{abstract}
Salidroside has been identified as one of the most potent compounds isolated from the plant Rhodiola rosea, and was found to have several important biological properties, including antioxidant and anti-inflammatory activity; however, its anticancer effects are poorly understood. Thus, the present study focused on evaluating the effects of purified salidroside on the growth of human breast cancer in vitro and in vivo, and on further investigating its possible molecular mechanisms. The human breast cancer cell line, MCF-7, was incubated with various concentrations of salidroside, and cell proliferation, colony formation, cell cycle distribution, apoptosis, migration and invasion were assayed by several in vitro approaches. As a result, it was found that salidroside treatment significantly inhibited cell proliferation, colony formation, migration and invasion, as well as induced cell apoptosis and cell cycle arrest at the G0/G1 phase in vitro. In addition, we also evaluated the effect of salidroside on tumor growth in a nude mouse model, and found that salidroside treatment significantly suppressed tumor growth in vivo. We also further disclosed that salidroside treatment significantly inhibited the intracellular reactive oxygen species (ROS) formation and MAPK pathway activation, which may contribute to the inhibition of tumor growth of breast cancer and reduction of oxidative stress. In conclusion, these findings suggest that salidroside may be a promising candidate target for the prevention and treatment of human breast cancer.
\end{abstract}

\section{Introduction}

Breast cancer is the most common malignancy and is a leading cause of cancer-related deaths among women, in developed and developing countries (1). Advanced and metastatic breast cancer is particularly difficult to treat, causing the secondleading cause of cancer-related deaths in women (2). In 2013, $\sim 230,000$ women were estimated to be diagnosed with breast cancer and more than 40,000 succumbed to the disease in

Correspondence to: Professor Ye Du, Department of Breast Surgery, The First Hospital, Jilin University, 71 Xinmin Street, Chaoyang, Changchun, Jilin 130021, P.R. China

E-mail: duye2134@126.com

Key words: breast cancer, salidroside, tumor growth the USA (3). Although several types of treatment, including surgery, radiotherapy, chemotherapy and hormone therapy, have been designed to treat breast cancer, the success to date is limited (4). Among the various types of therapy, systemic chemotherapy is the main treatment for cancer. However, chemotherapeutic drugs for breast cancer usually have variable effectiveness with high toxicity to normal tissues, and breast tumors often develop metastasis and drug resistance (5). Therefore, searching for effective regimens with minimal side effects remains the top priority in breast cancer research.

It has been demonstrated that plant-derived anticancer drugs are much more effective and have minimal side effects when compared to synthetic drugs $(6,7)$. Classic examples of plant-derived anticancer drugs that are currently in clinical use include vinblastine, vincristine, paclitaxel, camptothecin, as well as Rhodiola rosea (6-10).

Salidroside ( $p$-hydroxyphenethyl- $\beta$-D-glucoside)(chemical structure shown in Fig. 1A) is the main active ingredient found in Rhodiola rosea L, and has displayed many pharmacological properties including anti-aging, anti-fatigue, antioxidant, antiviral and anti-inflammatory effects, as well as neuroprotective and cardiovascular protective effects (11-18). Recently, salidroside has been found to inhibit cell proliferation, and induce cell apoptosis in lung (19) and bladder cancer (20), neuroblastoma (21) and glioma (22) in vitro. Regarding breast cancer, despite a recent report showing that salidroside induces cell cycle arrest and apoptosis in human breast cancer cells (23), the potential role of salidroside against breast cancer cell migration and invasion in vitro, and tumor growth ability in vivo have not been fully clarified. More significantly, the underlying mechanism of the anticancer effect of salidroside remains largely unknown.

The aim of the present study was to evaluate the potency of salidroside in inhibiting breast cancer cell proliferation, colony formation, migration and invasion in vitro and to reveal the underlying molecular mechanisms involved in the anticancer effects. In addition, tumor growth ability in nude mice was detected to define the salidroside treatment effect on tumorigenesis in vivo.

\section{Materials and methods}

Reagents and antibodies. Salidroside (purity, >99\%) was purchased from the National Institute of Pharmaceutical and 
Biological Products (Beijing, China). Salidroside was dissolved in water and filtered through a $0.22-\mu \mathrm{m}$ filter before use. Propidium iodide (PI) and 3-(4,5-dimethylthiazol-2-yl)-2,5 diphenyltetrazolium bromide (MTT) were purchased from Sigma-Aldrich (St. Louis, MO, USA). Stock solutions of PI and MTT were prepared by dissolving $1 \mathrm{mg}$ of each compound in $1 \mathrm{ml}$ of phosphate-buffered saline (PBS). The solution was protected from light, stored at $4^{\circ} \mathrm{C}$, and used within 1 month.

For western blot analysis, the following antibodies were used: mouse monoclonal anti-human $\beta$-actin (Sigma-Aldrich), mouse monoclonal anti-human $\mathrm{Bcl} 2$, mouse monoclonal anti-human Bax, mouse monoclonal anti-human p21, mouse monoclonal anti-human cyclin D1, mouse monoclonal anti-human cyclin D3, mouse monoclonal anti-human matrix metalloproteinase (MMP)-2, mouse monoclonal anti-human MMP-9, mouse monoclonal anti-human p38MAPK, mouse monoclonal anti-human phosphorylated (p)-p38MAPK, mouse monoclonal anti-human c-Jun N-terminal kinase (JNK), mouse monoclonal anti-human p-JNK, mouse monoclonal anti-human ERK1/2 and mouse monoclonal antihuman p-ERK1/2 (all from Santa Cruz Biotechnology, Santa Cruz, CA, USA). Secondary antibody HRP-conjugated goat anti-mouse IgG was purchased from Amersham Biosciences (Uppsala, Sweden).

Cell lines and culture. Human breast cancer cell line, MCF-7, was purchased from the Cell Bank of the Type Culture Collection of the Chinese Academy of Sciences, Shanghai Institute of Cell Biology, Chinese Academy of Sciences, (Shanghai, China) and cultured in Dulbecco's modified Eagle's medium (DMEM) supplemented with 10\% fetal bovine serum (FBS) (both from Gibco-BRL, Gaithersburg, MD, USA), $100 \mathrm{U} / \mathrm{ml}$ of penicillin and $0.1 \mathrm{mg} / \mathrm{ml}$ of streptomycin at $37^{\circ} \mathrm{C}$ in a humidified atmosphere of $5 \% \mathrm{CO}_{2}$.

MTT assay. Cell proliferation was determined by MTT assay as previously described (24). Briefly, $1 \times 10^{4}$ cells/well were cultivated in $100 \mu \mathrm{l}$ of culture medium in 96-well flat-bottomed plates (Corning Inc., Corning, NY, USA) and incubated with different concentrations of salidroside $(0.01-50 \mu \mathrm{M})$ for $72 \mathrm{~h}$, followed by the addition of $10 \mu \mathrm{l}$ of MTT solution ( $5 \mathrm{mg} / \mathrm{ml}$, dissolved in PBS; Sigma-Aldrich). After a 4-h incubation, $100 \mu \mathrm{l}$ of SDS $(10 \%$, w/v, dissolved in $0.01 \mathrm{M} \mathrm{HCl}$; Sigma-Aldrich) was added and mixed thoroughly to dissolve the formazan crystals at $37^{\circ} \mathrm{C}$. After shaking the plates for $10 \mathrm{~min}$, the absorbance was read at $570 \mathrm{~nm}$ in an ELISA plate reader (Molecular Devices Corp., Sunnyvale, CA, USA).

Colony formation assay. Cells were seeded in 6-well culture plates at a density of $1 \times 10^{4}$ cells/well. After being cultured for $24 \mathrm{~h}$, the cells were treated with different concentrations of salidroside $(0,5,20$ and $40 \mu \mathrm{M})$. After 14 days, the cells were washed, fixed by paraformaldehyde, and stained with Giemsa for $10 \mathrm{~min}$. Then extra Giemsa was washed 3 times by $\mathrm{ddH}_{2} \mathrm{O}$, and the colonies were photographed using a digital camera. The visible colonies in each group were counted.

Quantitative analysis of apoptotic cells by Annexin V/PI staining. Apoptotic cell death induced by salidroside was quantified by flow cytometry using the Annexin V-fluorescein isothiocyanate (FITC) kit following the manufacturer's instructions. Briefly, the cells were plated at a density of $3 \times 10^{5}$ cells/well in a 6 -well plate and incubated with different concentrations of salidroside $(0,5,20$ and $40 \mu \mathrm{M})$ for $48 \mathrm{~h}$. Floating cells as well as residual attached cells were collected and washed twice with PBS. The cell pellets were resuspended in $500 \mu 1$ of $1 \mathrm{X}$ binding buffer at a concentration of $1 \times 10^{6}$ cells $/ \mathrm{ml}$. Five microliters of Annexin V-FITC and PI was added to the cell suspension for $10 \mathrm{~min}$ at room temperature, stained samples were examined using a Coulter Epics XL flow cytometer (Beckman Coulter, Miami, FL, USA), and the data were analyzed using CellQuest software (BD Biosciences, San Jose, CA, USA). Experiments were performed in triplicate. In addition, we also detected Bax and Bcl-2 expression by western blotting as an additional indicator of apoptosis.

Measurement of caspase activity. Caspase- $3,-8$ and -9 activity was measured using the Caspase Colorimetric Assay kit (Millipore Corporation, Billerica, MA, USA) according to the manufacturer's instructions. Briefly, the cells after treatment for $48 \mathrm{~h}$ were harvested and lysed in lysis buffer on ice for $10 \mathrm{~min}$ and then centrifuged at $10,000 \mathrm{x}$ g for $1 \mathrm{~min}$. After centrifugation, the supernatants were incubated with caspase- $3,-8$ and -9 substrates in reaction buffer. Samples were seeded into a 96-well flat-bottom microplate at $37^{\circ} \mathrm{C}$ for $1 \mathrm{~h}$. Samples were analyzed at $405 \mathrm{~nm}$ in a microplate reader (Thermo Fisher Scientific Inc., Waltham, MA, USA). The relative caspase activity of the untreated group ( $0 \mu \mathrm{M}$ salidroside treatment) was taken as 100. Each assay was conducted in triplicate.

Cell cycle analysis. To determine the cell cycle distribution, the cells were plated in $60-\mathrm{mm}$ dishes and treated with different concentrations of salidroside $(0,5,20$ and $40 \mu \mathrm{M})$ for $48 \mathrm{~h}$. After treatment, the cells were collected by trypsinization, fixed in $70 \%$ ethanol, and kept at $-20^{\circ} \mathrm{C}$ overnight for fixation. Cells were washed twice with PBS, and then resuspended in $1 \mathrm{ml}$ of PBS containing RNase (100 $\mu \mathrm{g} / \mathrm{ml})$ and PI $(40 \mu \mathrm{g} / \mathrm{ml})$ in the dark for $30 \mathrm{~min}$ at room temperature. The distribution of cells in the cell cycle phases was analyzed from the DNA histogram with a FACS Caliber flow cytometer (Becton-Dickinson, San Jose, CA, USA). The data were analyzed using CellQuest software (BD Biosciences). Furthermore, we also detected p21, cyclin D1 and cyclin D3 expression by western blotting as an additional indicator of cell cycle arrest.

Transwell migration and invasion assays. To assess the effect of salidroside on cell migration and invasion, the migration and invasion assays were performed using Transwell insert chambers (Corning Inc.). For the migration assay, the cells were incubated with different concentrations of salidroside $(0,5,20$ and $40 \mu \mathrm{M})$ for $48 \mathrm{~h}$. After treatment, a total of $1 \times 10^{5}$ cells were plated into the upper chamber in serum-free DMEM. Medium containing 20\% FBS in the lower chamber served as chemoattractant. After being cultured for $24 \mathrm{~h}$, the media were removed from the upper chamber by wiping with a cotton swab and cells that migrated to the lower surface of the filter were fixed in $70 \%$ ethanol for 30 min followed by staining with $0.2 \%$ crystal violet for $10 \mathrm{~min}$. Cell migration was counted by counting five random fields per filter under a light microscope (Olympus, Tokyo, Japan). 
For the invasion assay, after treatment, $3 \times 10^{5}$ cells were incubated in the upper chambers pre-coated with Matrigel (BD Biosciences) in serum-free DMEM, and the subsequent steps were consistent with the migration assay. The number of cells invading the Matrigel was counted in five randomly selected fields using a light microscope (Olympus). All experiments were performed in triplicate.

Measurement of intracellular reactive oxygen species (ROS). Intracellular changes in ROS were determined using the MGT Live Cell Fluorescent ROS Detection kit (MGT, Inc., USA) as described previously (24). Briefly, cells were plated in a 96-well plate $\left(25 \times 10^{3}\right.$ cells/well) and were treated with different concentrations of salidroside $(0,5,20$ and $40 \mu \mathrm{M})$ for $48 \mathrm{~h}$. After treatment, the cells were further incubated with $20 \mu \mathrm{M}$ 2',7'-dichlorofluorescein diacetate in Hank's balanced salt solution (HBSS) at $37^{\circ} \mathrm{C}$ for $30 \mathrm{~min}$ in the dark. Subsequently, the cells were harvested and washed with HBSS and analyzed for DCF fluorescence using a Synergy HT Multi-Mode microplate reader (BioTek Instruments, San Jose, CA, USA).

Western blot analysis. The cells were collected by trypsinization and lysed in radioimmunoprecipitation assay lysis buffer (Sigma-Aldrich) with the addition of protease inhibitors (Roche) and phosphatase inhibitors (Sigma-Aldrich) for $30 \mathrm{~min}$ on ice. Then the homogenates were centrifuged at 14,000 rpm at $4^{\circ} \mathrm{C}$ for $30 \mathrm{~min}$ to remove insoluble material, and the supernatants were collected for protein concentration determination using the BCA protein assay kit (Sigma-Aldrich). The cell extracts ( $20 \mu \mathrm{g}$ of protein) were separated on a sodium dodecyl sulfate-polyacrylamide electrophoretic gel (SDS-PAGE) and transferred to nitrocellulose membranes. The membranes were blocked with 5\% dry milk in PBS and incubated with the primary antibodies. After incubation with primary antibodies, the membranes were washed in PBS and incubated with secondary horseradish peroxidase-coupled goat anti-mouse antibodies. The proteins were detected and protein bands were visualized with enhanced chemiluminescence reagent (ECL; Sigma-Aldrich). The integrated density value (IDV) was analyzed with a computerized image analysis system (Fluor Chen 2.0) and normalized with that of $\beta$-actin.

Tumor xenografts in nude mice. Female BALB/c nude mice at 6-7 weeks of age were obtained from the Experimental Animal Center of Jilin University (Changchun, China) and were housed under standard conditions. MCF-7 cells were trypsinized and washed with PBS and suspended in DMEMfree serum. A total of $2 \times 10^{6}$ cells were injected into the flanks of nude mice. Tumor growth was measured every 7 days, and tumor volume was estimated as length $\mathrm{x}$ width $\mathrm{x}$ height $x 0.5236$. When tumors grew to an average volume of $100 \mathrm{~mm}^{3}$, the mice were divided randomly into 2 groups (10 mice/group). The control group received $1 \%$ PBS in deionized water. The treatment group was treated with salidroside $(50 \mathrm{mg} / \mathrm{kg}$ body weight) intraperitoneally on alternate days for 3 weeks. The doses were selected based on a previous experiment (22). The tumor size was measured using a caliper on days 7, 14 and 21 of treatment. On day 21, the animals were sacrificed using chloroform, and tumor tissues were isolated and weighed. In addition, spleen tissues were collected and cultured for a sple- nocyte surveillance study to assay splenocyte proliferation as previously described (25). All procedures were in agreement with Jilin University Guide for the Care and Use of Laboratory Animals and were approved by the Animal Care and Use Committee, Jilin University (Changchun, China).

Statistical analysis. All experiments were performed at least three independent times, and the results are expressed as the mean \pm standard deviation (SD). For statistical comparison of quantitative data between groups, analysis of variance (ANOVA) or Student's t-test was performed. All statistical analyses were performed using the GraphPad Prism version 5.01 (GraphPad Software, San Diego, CA, USA) and the SPSS software (version 16.0; SPSS Inc., Chicago, IL, USA) for Windows ${ }^{\circledR}$. P-values $<0.05$ were considered to be statistically significant.

\section{Results}

Salidroside inhibits proliferation and colony formation in MCF-7 cells. To determine the cytotoxic effects of salidroside on MCF-7 cells, MTT assay was carried out. It was found that salidroside significantly inhibited the viability of the MCF-7 cells in a dose-dependent manner (Fig. 1B). In the MCF-7 cells, as shown in Fig. 1B, a significant inhibitory effect was observed at $1 \mu \mathrm{M}$, which reached a maximum level at $50 \mu \mathrm{M}$. The $\mathrm{IC}_{50}$ value (the effective dose that inhibits $50 \%$ of growth) for the treatment of MCF-7 cells by salidroside was 19.48 $(\mathrm{P}<0.05)$. Based on the results, we chose concentrations of 5, $20\left(\mathrm{IC}_{50}\right)$ and $40 \mu \mathrm{M}\left(2 \mathrm{x} \mathrm{IC}_{50}\right)$ salidroside for further treatments throughout the study.

Next, the effect of salidroside on the cell colony formation of MCF-7 cells was also analyzed. As shown in Fig. 1C, salidroside significantly inhibited the colony formation of MCF-7 cells in a dose-dependent manner $(\mathrm{P}<0.05)$.

Salidroside induces apoptosis in MCF-7 cells. To determine whether the growth inhibitory effect of salidroside is associated with the induction of apoptotic cell death, flow cytometry was performed. MCF-7 cells were exposed to various concentrations $(0,5,20$ and $40 \mu \mathrm{M})$ of salidroside for $48 \mathrm{~h}$ and analyzed by flow cytometry. As shown in Fig. 2A, we observed a dose-dependent increase in apoptotic cells in the presence of salidroside in MCF-7 cells.

To examine the contribution of caspases in the salidrosideinduced apoptosis, the role of caspase-3, -8 and -9 was investigated. The results demonstrated that treatment of MCF-7 cells with salidroside resulted in a significant increase in the activities of caspase-3, -8 and -9 in a dose-dependent manner (Fig. 2B-D).

To determine the potential mechanism involved in the effect on cell apoptosis by salidroside, expression levels of apoptosisrelated proteins, Bax and $\mathrm{Bcl}-2$, were examined by western blot analysis. Western blot analysis displayed a significant upregulation of Bax expression, and downregulation of Bcl-2 expression in the MCF-7 cells following treatment with salidroside in a dose-dependent manner $(\mathrm{P}<0.05$, Fig. $2 \mathrm{E}$ and $\mathrm{F})$.

Salidroside induces G0/G1 phase cell cycle arrest in MCF-7 cell. To determine whether the growth inhibitory effect of salidroside is associated with cell cycle arrest, flow cytom- 

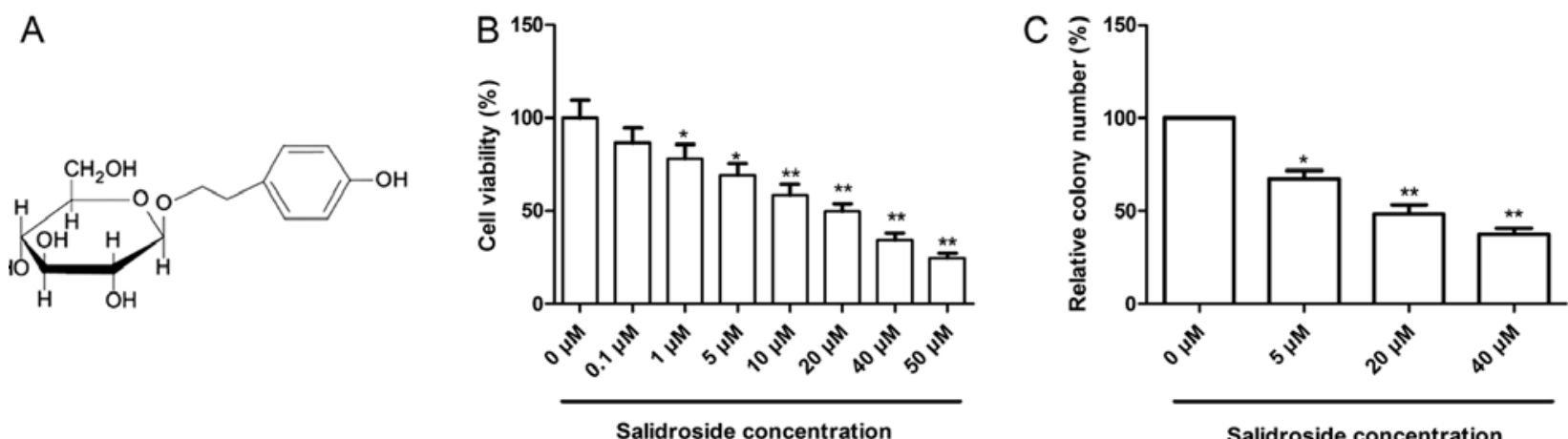

Figure 1. Salidroside inhibits the proliferation and colony formation of MCF-7 cells. (A) The chemical structure of salidroside. (B) MTT was performed to analyze the effect of salidroside on the cell proliferation of MCF-7 cells after treatment with different concentrations of salidroside. (C) The effect of salidroside on the colony formation of the MCF-7 cells was determined after treatment with different concentrations of salidroside. Data are derived from three independent experiments. ${ }^{*} \mathrm{P}<0.05,{ }^{* * *} \mathrm{P}<0.01$ vs. the untreated cells $(0 \mu \mathrm{M})$.
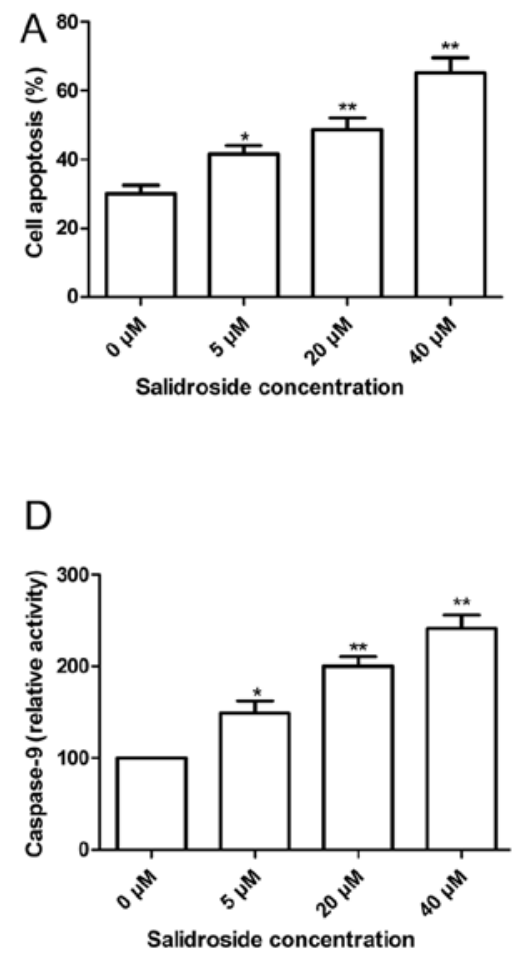

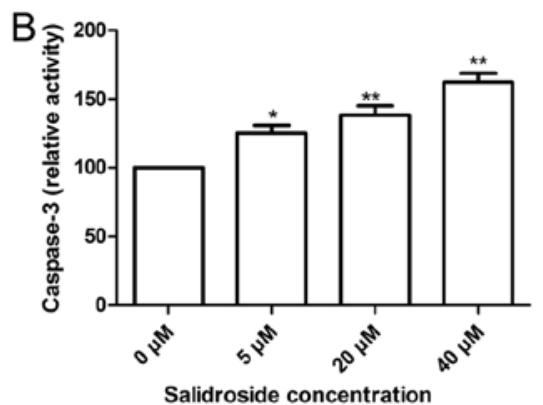

E
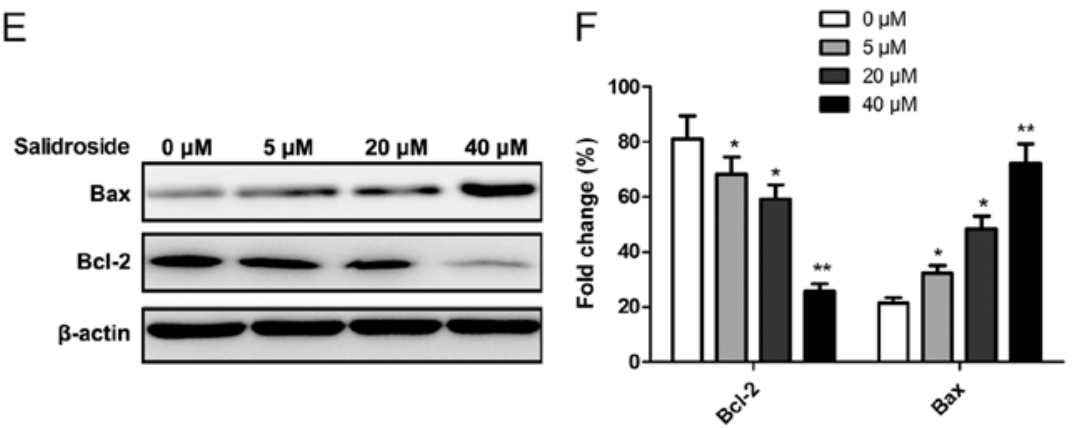

Figure 2. Salidroside induces the cell apoptosis of MCF-7 cells. (A) Cell apoptosis was assayed after treatment with different concentrations of salidroside. (B) Caspase-3, (C) caspase-8 and (D) caspase-9 activities were determined by ELISA after treatment with different concentrations of salidroside. (E) Western blot analysis of Bax-2 and Bcl-2 protein expression after treatment with different concentrations of salidroside. $\beta$-actin was used as an internal control. (F) Relative quantification of Bax and Bcl-2 protein by densitometric analysis. ${ }^{*} \mathrm{P}<0.05,{ }^{* *} \mathrm{P}<0.01$ vs. the untreated cells $(0 \mu \mathrm{M})$.

etry was performed to determine the cell cycle distribution. The MCF7 cells were treated with various concentrations of salidroside $(0,5,20$ and $40 \mu \mathrm{M})$ for $48 \mathrm{~h}$. It was found that salidroside significantly increased the percentage of cells in the $\mathrm{G} 0 / \mathrm{G} 1$ phase in a dose-dependent manner $(\mathrm{P}<0.05)$, while the percentage of cells in the $\mathrm{S}$ phase significantly decreased following salidroside treatment $(\mathrm{P}<0.05$, Fig. $3 \mathrm{~A}$ and $\mathrm{B})$.

Next, we analyzed the effects of salidroside on the expression of cell cycle-associated proteins, such as cyclin D1, cyclin D3 and p21 by western blotting. As shown in Fig. 3C and $\mathrm{D}$, salidroside treatment markedly increased p21 expression, while cyclin D1 and cyclin D3 expression was significantly decreased in the MCF-7 cells in dose-dependent manner $(\mathrm{P}<0.05)$.

Salidroside inhibits cell migration and invasion in MCF-7 cells. We were particularly interested in whether salidroside affects cell vitality, demonstrated by migration or invasion activity. Thus, cell migration and invasion assays were performed by Transwell assay. It was found that salidroside significantly decreased the migration of MCF-7 cells in a dose-dependent manner $(\mathrm{P}<0.05$, Fig. 4A). The ability of salidroside to reduce the invasiveness of MCF-7 cells was further investigated. Transwell matrix penetration (coated with Matrigel) assay showed that 

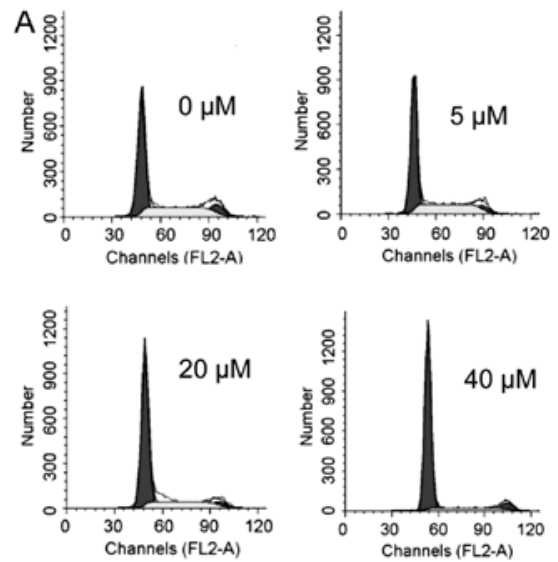

C

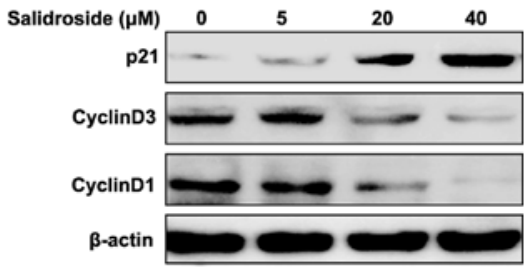

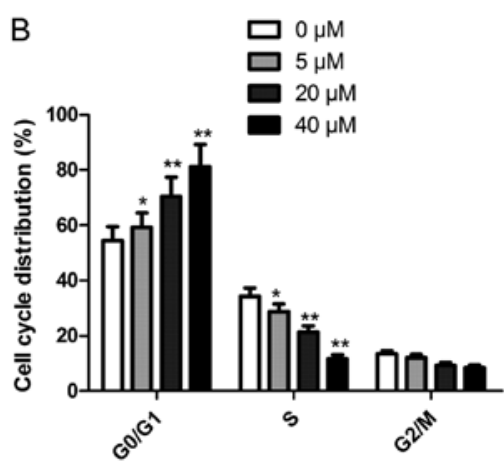

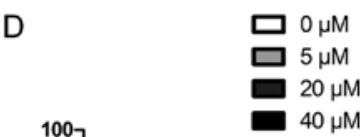

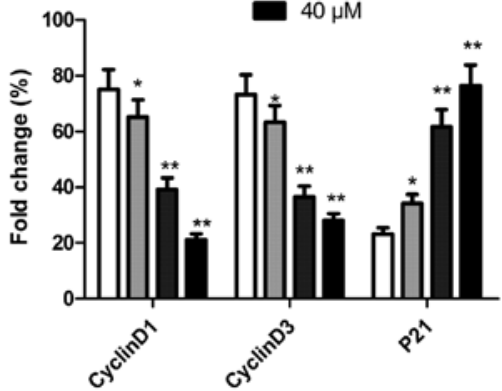

Figure 3. Salidroside induces cell cycle arrest at the G0/G1 phase. (A) Cell DNA content distribution in each phase after treatment with different concentrations of salidroside. (B) Percentage of cells distributed in each phase of the cell cycle after treatment with different concentrations of salidroside. (C) Western blot analysis of cyclin D1, cyclin D3 and p21 protein expression after treatment with different concentrations of salidroside. $\beta$-actin was used to an internal control. (D) Relative quantification of cyclin D1, cyclin D3 and p21 protein by densitometric analysis. ${ }^{*} \mathrm{P}<0.05,{ }^{* *} \mathrm{P}<0.01$ vs. untreated cells $(0 \mu \mathrm{M})$.

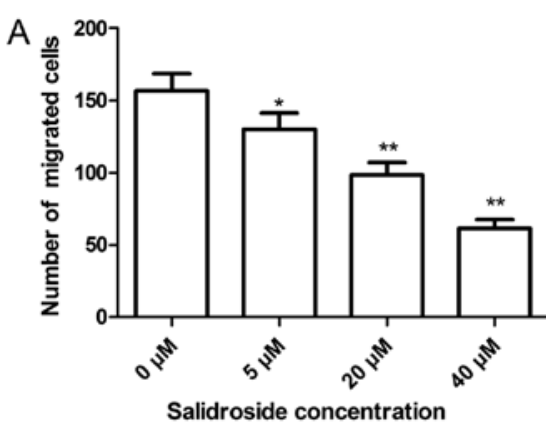

C

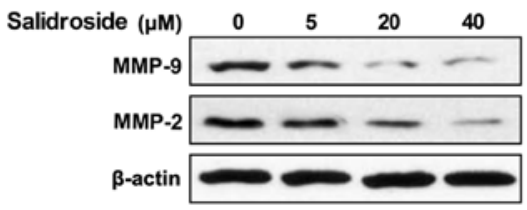

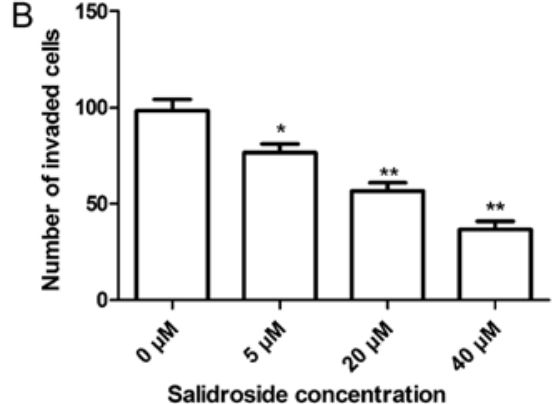

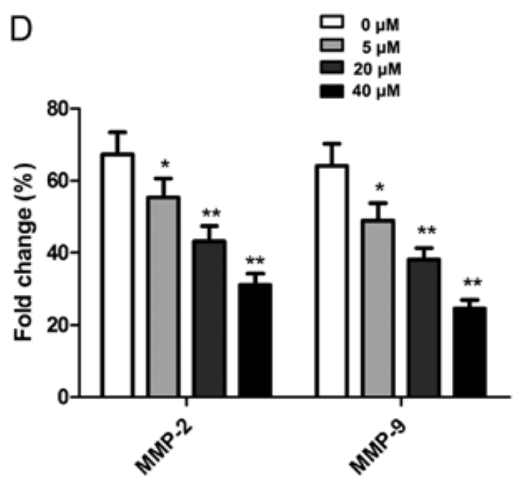

Figure 4. Salidroside inhibits the migration and invasion of MCF-7 cells. (A) The number of migrated cells was determined using the Transwell assay (without Matrigel) after treatment with different concentrations of salidroside. (B) The number of invaded cells was determined using the Transwell matrix penetration assay (with Matrigel) after treatment with different concentrations of salidroside. (C) Western blot analysis of MMP-2 and MMP-9 protein expression after treatment with different concentrations of salidroside. $\beta$-actin was used as an internal control. (D) Relative quantification of MMP-2 and MMP-9 protein by densitometric analysis. ${ }^{*} \mathrm{P}<0.05,{ }^{* *} \mathrm{P}<0.01$ vs. untreated cells $(0 \mu \mathrm{M})$. MMP, matrix metalloproteinase. 
A

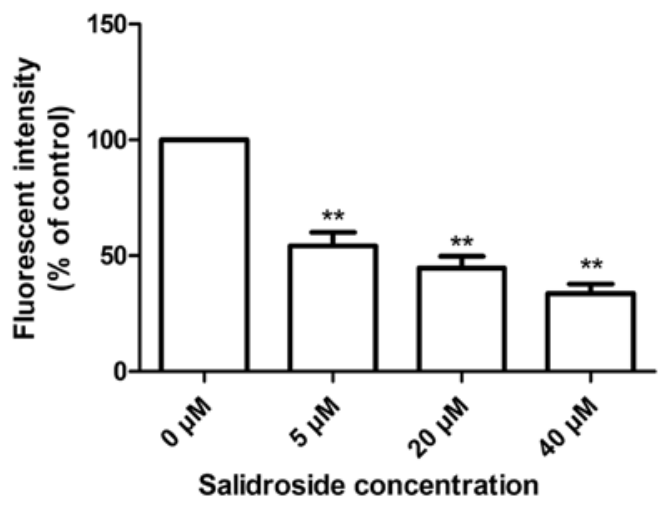

B

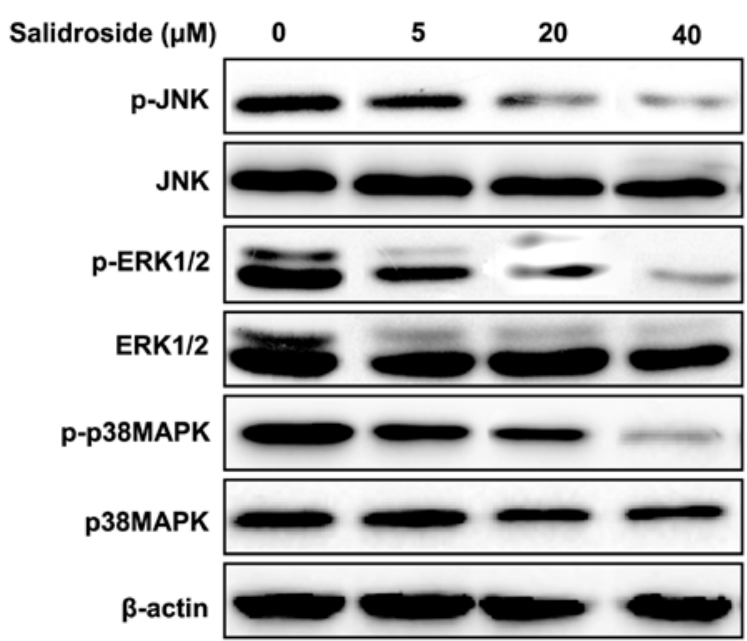

Figure 5. Effects of salidroside on the intracellular ROS formation and MAPK signaling pathway in MCF-7 cells. (A) Intracellular ROS levels were measured by the fluorescent probe DCFH-DA after a 48-h treatment with salidroside. The fluorescence intensity was expressed as the percentage of the control. (B) MCF-7 cells were treated with various concentrations of salidroside for $12 \mathrm{~h}$. The phosphorylation of p38MAPK, ERK1/2 and JNK was analyzed by western blotting method. $\beta$-actin was used as an internal control. ${ }^{*} \mathrm{P}<0.05,{ }^{* *} \mathrm{P}<0.01$ vs. untreated cells $(0 \mu \mathrm{M})$. ROS, reactive oxygen species; JNK, c-Jun N-terminal kinase.

salidroside markedly reduced the invasiveness of MCF-7 cells in dose-dependent manner $(\mathrm{P}<0.05$, Fig. 4B).

To determine the potential mechanism involved in the effect on cell migration and invasion by salidroside, expression of MMP-2 and MMP-9 protein was determined by western blotting. Western blot analysis showed that salidroside significantly reduced MMP-2 and MMP-9 expression in the MCF-7 cells in a dose-dependent manner ( $\mathrm{P}<0.05$, Fig. 4C and D).

Effect of salidroside on the intracellular ROS formation and MAPK signaling pathway in MCF-7 cells. ROS are produced particularly when cells undergo chemical or environmental stress and could be one of the factors leading to cell apoptosis. Therefore, the ROS level after a 48-h treatment with salidroside was examined in the breast cancer cells by the fluorescent probe DCFH-DA. It was found that salidroside treatment significantly reduced the intracellular ROS level in the MCF-7 cells in a dose-dependent manner (Fig. 5A).

It is well known that the MAPK signaling pathway plays a crucial role in cell proliferation and survival in various cancers. In addition, ROS production has been shown to be coupled with the sustained activation of the MAPK signaling pathway for a variety of cellular effects (26). Therefore, in the present study, we next evaluated the effect of salidroside on several key downstream molecules involved in the MAPK signaling pathway. Measurements of the phosphorylation/activation pattern of p38MAPK, ERK1/2 and JNK were performed by western blotting $12 \mathrm{~h}$ after treatment with salidroside. It was found that salidroside treatment resulted in a marked reduction in phosphorylated p38MAPK, ERK1/2 and JNK in the MCF-7 cells in a dose-dependent manner, without altering the total protein levels of p38MAPK, ERK1/2 and JNK at different concentrations of salidroside (Fig. 5B).

Salidroside suppresses tumor growth in a nude mouse model. We aimed to ascertain whether salidroside treatment inhibits tumor growth in a xenograft tumor model. Tumor growth was monitored for 3 weeks. On day 21, mice were sacrificed, and the tumor weight was measured. It was found that tumor weight were significantly lower in the salidroside treatment group relative to the control group (untreated group) $(\mathrm{P}<0.05$, Fig. 6A and B). In addition, tumor volume was also determined at different times. The tumor volume in the salidroside treatment group was significantly diminished when compared with that in the control group (Fig. 6A and C). In addition, we employed MTT assays in modulating splenocyte proliferation to demonstrate the antitumor activities of salidroside in vivo. It was found that splenocyte cell proliferation in the salidroside treatment group significantly decreased relative to the control group $(\mathrm{P}<0.05$, Fig. 6D). These data suggest that salidroside treatment suppresses the tumor growth of breast cancer in vivo.

\section{Discussion}

Although recent advances in diagnosis and treatment, breast cancer mortality rates remain high. Conventional anticancer chemotherapy are associated with occurrence of side effects induced by the non-specific targeting of both normal and cancer cells (27). Therefore, development of novel agents for the prevention and treatment of human breast cancer is urgently needed. As a valuable source for novel chemotherapeutic agents, natural plant compounds exhibit effective anticancer activities with few side effect $(6,28)$. Salidroside is a phenylpropanoid glycoside isolated from a popular traditional Chinese medicinal plant, Rhodiola rosea $\mathrm{L}$, and has been proven to induce cell apoptosis and cell cycle arrest in breast cancer cells (23), while the effect of salidroside on cancer cell migration and invasion has not yet been reported. The present study demonstrated for the first time that salidroside treatment effectively inhibited the migration and invasion of MCF-7 cells, and suppressed the tumor growth of breast cancer in vivo.

Growing evidence has shown that inhibition of antiapoptotic members or activation of pro-apoptotic members of the Bcl-2 family usually leads to an altered mitochondrial membrane permeability, which allows the release of cyto- 
A

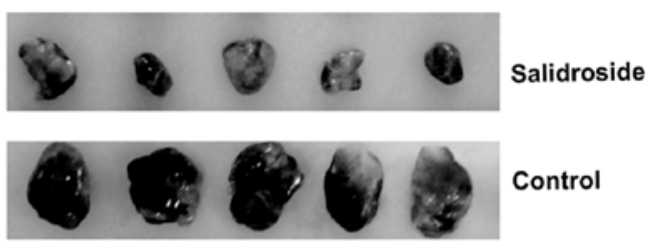

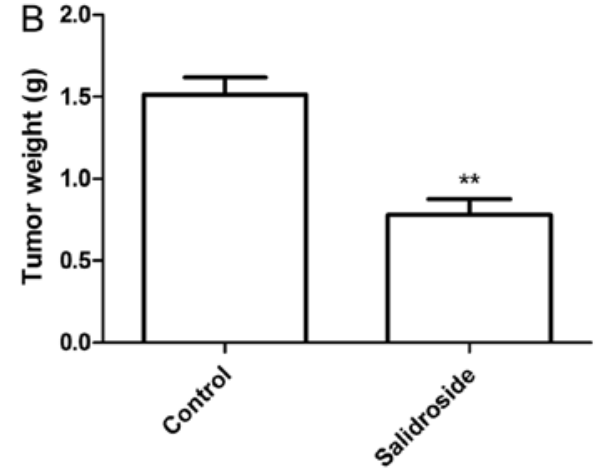
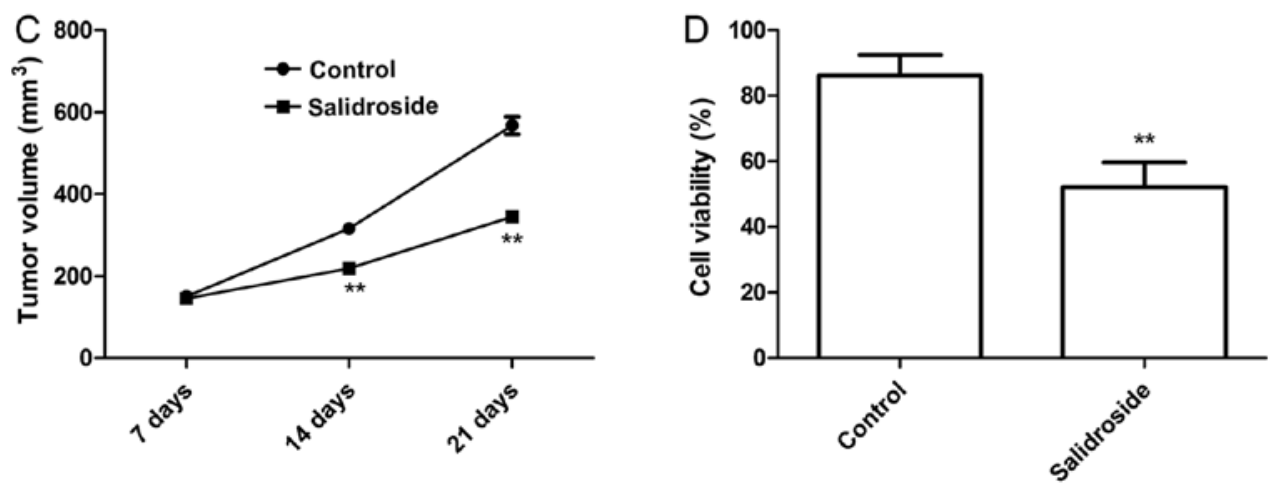

Figure 6. Antitumor activity of salidroside in BALB/c mice bearing MCF-7 cell-derived tumors. (A) Graphs of the tumor tissue from the different groups. (B) Tumor weight of each group was measured on day 21. (C) Tumor volume in each group was determined on days 7, 14 and 21. (D) MTT assay of proliferation of splenocytes from the mice. ${ }^{*} \mathrm{P}<0.05,{ }^{* *} \mathrm{P}<0.01$ vs. the control.

chrome $c$ into the cytosol and the subsequent activation of caspase-3 and -9 , leading to apoptotic cell death (29). Caspases (a family of proteases) are one of the essential executioners of apoptosis, and their cleavage and subsequent activation are considered as the primary hallmarks of apoptosis (30). To understand the potential antitumor mechanisms, the relative levels of expression of Bcl-2 and Bax following salidroside treatment were detected by western blot analysis, and the caspase-3, -8 and -9 activities were measured by a caspase kit. The present study showed that salidroside downregulated the expression of Bcl-2, and upregulated the expression of Bax, as well as increased caspase-3, -8 and -9 activity in the MCF-7 cells in a concentration-dependent manner $(\mathrm{P}<0.05)$. These results imply that salidroside induced cell apoptosis by inhibiting Bcl-2 expression, leading to activation of caspase- $-3,-8$ and -9 .

MMPs are the main family of proteolytic enzymes that facilitate tumor cell migration by degrading the basement membrane, and other components of the extracellular matrix (ECM) play a key role in tumor cell invasion, migration and tumor angiogenesis $(31,32)$. MMP-2 and MMP-9 are important members of the MMP family, and downregulation of the expression of MMP-2 and MMP-9 contributes to inhibit cancer cell invasion and metastasis $(33,34)$. Recently a report showed that salidroside significantly suppressed MMP-2 and MMP-9 activity, and increased tissue inhibitor of metalloproteinase-2 (TIMP-2) expression in a dose-dependent manner in HT1080 cells (35). Consistent with this result, our present study demonstrated that salidroside decreased MMP-2 and MMP-9 activity in MCF-7 cells. Thus, salidroside may suppress MMP-2 and MMP-9 activity to reduce the metastatic capabilities of MCF-7 cells.
The MAPK signaling cascade, including ERK1/2, JNK, and $\mathrm{p} 38$, has been reported to play a crucial role in signal transduction and mediates cellular proliferation, differentiation, inflammation and apoptosis (36). It was known that a high level of ROS destroys the integrity of the plasma membrane, affects the dynamics of the actin cytoskeleton, and causes DNA damage, cumulatively known as oxidative stress, leading to disruptions in normal mechanisms of cellular signaling $(37,38)$. ROS production has been shown to be coupled with the sustained activation of the ERK signaling pathway for a variety of cellular effects, such as apoptosis and phagocytosis (26). Recently, Wang et al reported that salidroside inhibited the intracellular ROS formation and phosphorylation of p38 in A549 cells in a dose-dependent manner (19). Panossian et al found that salidroside reduced the phosphorylation of JNK to fight against fatigue and stress in rabbits (39). Sun et al showed that salidroside treatment significantly decreased the intracellular ROS level and inhibited phosphorylation of ERK1/2 expression in HT1080 cells (35). Consistent with these results, we showed that salidroside treatment inhibited the intracellular ROS formation, and suppressed phosphorylation of p38, JNK and ERK1/2 expression in MCF-7 cells in a dose-dependent manner. These findings suggest that salidroside treatment inhibited human breast cancer growth, which may be due to its downregulation of intracellular ROS formation and attenuation of the activation of the MAPK family signaling pathway.

In conclusion, the findings of the present study provide evidence that salidroside significantly inhibits breast cell proliferation, migration and invasion, and induces cancer cell apoptosis in vitro, as well as suppresses tumor growth in vivo. 
In addition, salidroside treatment significantly inhibited the intracellular ROS formation and MAPK pathway activation, which may contribute to the inhibition of tumor growth and decreased oxidative stress. Thus, salidroside may be a promising natural compound for human breast cancer chemoprevention or chemotherapy.

\section{References}

1. Siegel R, Naishadham D and Jemal A: Cancer statistics, 2013. CA Cancer J Clin 63: 11-30, 2013.

2. Clark O, Botrel TE, Paladini L and Ferreira MB: Targeted therapy in triple-negative metastatic breast cancer: a systematic review and meta-analysis. Core Evid 9: 1-11, 2014.

3. DeSantis C, Ma J, Bryan L and Jemal A: Breast cancer statistics, 2013. CA Cancer J Clin 64: 52-62, 2014.

4. Murphy IG, Dillon MF, Doherty AO, McDermott EW, Kelly G, O'Higgins N and Hill AD: Analysis of patients with false negative mammography and symptomatic breast carcinoma. J Surg Oncol 96: 457-463, 2007.

5. Nicolin V, Fancellu G and Valentini R: Effect of tanshinone II on cell growth of breast cancer cell line type MCF-7 and MD-MB-231. Ital J Anat Embryol 119: 38-43, 2014.

6. Butler MS: The role of natural product chemistry in drug discovery. J Nat Prod 67: 2141-2153, 2004.

7. Paterson I and Anderson EA: Chemistry. The renaissance of natural products as drug candidates. Science 310: 451-453, 2005

8. Maison W: Natural products research: renaissance with strengthened integration of biology and chemistry. Angew Chem Int Ed Engl 45: 3000-3002, 2006.

9. Xu YC, Wang HX, Tang L, Ma Y and Zhang FC: A systematic review of vinorelbine for the treatment of breast cancer. Breast J 19: 180-188, 2013.

10. Loo WT, Jin LJ, Chow LW, Cheung MN and Wang M: Rhodiola algida improves chemotherapy-induced oral mucositis in breast cancer patients. Expert Opin Investig Drugs 19 (Suppl 1): S91-S100, 2010.

11. Xu MC, Shi HM, Wang H and Gao XF: Salidroside protects against hydrogen peroxide-induced injury in HUVECs via the regulation of REDD1 and mTOR activation. Mol Med Rep 8 : 147-153, 2013.

12. Zhu Y, Shi YP, Wu D, Ji YJ, Wang X, Chen HL, Wu SS Huang DJ and Jiang W: Salidroside protects against hydrogen peroxide-induced injury in cardiac $\mathrm{H} 9 \mathrm{c} 2$ cells via PI3K-A kt dependent pathway. DNA Cell Biol 30: 809-819, 2011.

13. Tan CB, Gao M, Xu WR, Yang XY, Zhu XM and Du GH: Protective effects of salidroside on endothelial cell apoptosis induced by cobalt chloride. Biol Pharm Bull 32: 1359-1363, 2009.

14. Yuan Y, Wu SJ, Liu X and Zhang LL: Antioxidant effect of salidroside and its protective effect against furan-induced hepatocyte damage in mice. Food Funct 4: 763-769, 2013.

15. Chen X, Zhang Q, Cheng Q and Ding F: Protective effect of salidroside against $\mathrm{H}_{2} \mathrm{O}_{2}$-induced cell apoptosis in primary culture of rat hippocampal neurons. Mol Cell Biochem 332: 85-93, 2009

16. Wang H, Ding Y, Zhou J, Sun X and Wang S: The in vitro and in vivo antiviral effects of salidroside from Rhodiola rosea $\mathrm{L}$. against coxsackievirus B3. Phytomedicine 16: 146-155, 2009.

17. Chen X, Liu J, Gu X and Ding F: Salidroside attenuates glutamate-induced apoptotic cell death in primary cultured hippocampal neurons of rats. Brain Res 1238: 189-198, 2008.

18. Wu T, Zhou H, Jin Z, Bi S, Yang X, Yi D and Liu W: Cardioprotection of salidroside from ischemia/reperfusion injury by increasing $\mathrm{N}$-acetylglucosamine linkage to cellular proteins. Eur J Pharmacol 613: 93-99, 2009.

19. Wang J, Li JZ, Lu AX, Zhang KF and Li BJ: Anticancer effect of salidroside on A549 lung cancer cells through inhibition of oxidative stress and phospho-p38 expression. Oncol Lett 7: 1159-1164, 2014.

20. Liu Z, Li X, Simoneau AR, Jafari M and Zi X: Rhodiola rosea extracts and salidroside decrease the growth of bladder cancer cell lines via inhibition of the mTOR pathway and induction of autophagy. Mol Carcinog 51: 257-267, 2012.
21. Zhang L, Yu H, Sun Y, Lin X, Chen B, Tan C, Cao G and Wang Z: Protective effects of salidroside on hydrogen peroxide-induced apoptosis in SH-SY5Y human neuroblastoma cells. Eur J Pharmacol 564: 18-25, 2007.

22. Zhang Y, Yao Y, Wang H, Guo Y, Zhang H and Chen L: Effects of salidroside on glioma formation and growth inhibition together with improvement of tumor microenvironment. Chin J Cancer Res 25: 520-526, 2013.

23. Hu X, Zhang X, Qiu S, Yu D and Lin S: Salidroside induces cell-cycle arrest and apoptosis in human breast cancer cells. Biochem Biophys Res Commun 398: 62-67, 2010.

24. Hamzeloo-Moghadam M, Aghaei M, Fallahian F, et al: Britannin, a sesquiterpene lactone, inhibits proliferation and induces apoptosis through the mitochondrial signaling pathway in human breast cancer cells. Tumour Biol: Epub ahead of print Oct 24, 2014.

25. Abe S, Nishimoto $\mathrm{Y}$, Isu K, Ishii $\mathrm{T}$ and Goto T; Japanese Musculoskeletal Oncology Group: Preoperative cisplatin for initial treatment of limb osteosarcoma: its local effect and impact on prognosis. Cancer Chemother Pharmacol 50: 320-324, 2002.

26. Lee HB, Yu MR, Song JS and Ha H: Reactive oxygen species amplify protein kinase $\mathrm{C}$ signaling in high glucose-induced fibronectin expression by human peritoneal mesothelial cells. Kidney Int 65: 1170-1179, 2004.

27. Head J and Johnston SR: New targets for therapy in breast cancer: farnesyltransferase inhibitors. Breast Cancer Res 6: 262-268, 2004.

28. Newman DJ and Cragg GM: Natural products as sources of new drugs over the 30 years from 1981 to 2010. J Nat Prod 75: 311-335, 2012.

29. Green DR and Kroemer G: The pathophysiology of mitochondrial cell death. Science 305: 626-629, 2004.

30. Jänicke RU, Sprengart ML, Wati MR and Porter AG: Caspase-3 is required for DNA fragmentation and morphological changes associated with apoptosis. J Biol Chem 273: 9357-9360, 1998.

31. Rajoria S, Suriano R, George A, Shanmugam A, Schantz SP, Geliebter J and Tiwari RK: Estrogen induced metastatic modulators MMP-2 and MMP-9 are targets of 3,3'-diindolylmethane in thyroid cancer. PLoS One 6: e15879, 2011.

32. Yan L, Lin B, Gao L, Gao S, Liu C, Wang C, Wang Y, Zhang S and Iwamori M: Lewis (y) antigen overexpression increases the expression of MMP-2 and MMP-9 and invasion of human ovarian cancer cells. Int J Mol Sci 11: 4441-4452, 2010.

33. Braicu EI, Gasimli K, Richter R, et al; Tumor Bank Ovarian Cancer (TOC); German North Eastern Society for Gynecological Oncology (NOGGO): Role of serum VEGFA, TIMP2, MMP2 and MMP9 in monitoring response to adjuvant radiochemotherapy in patients with primary cervical cancer - results of a companion protocol of the randomized NOGGO-AGO phase III clinical trial. Anticancer Res 34: 385-391, 2014

34. Lai WW, Hsu SC, Chueh FS, Chen YY, Yang JS, Lin JP, Lien JC, Tsai $\mathrm{CH}$ and Chung JG: Quercetin inhibits migration and invasion of SAS human oral cancer cells through inhibition of $\mathrm{NF}-\kappa \mathrm{B}$ and matrix metalloproteinase-2/-9 signaling pathways. Anticancer Res 33: 1941-1950, 2013.

35. Sun C, Wang Z, Zheng Q and Zhang H: Salidroside inhibits migration and invasion of human fibrosarcoma HT1080 cells. Phytomedicine 19: 355-363, 2012.

36. Dhillon AS, Hagan S, Rath O and Kolch W: MAP kinase signalling pathways in cancer. Oncogene 26: 3279-3290, 2007.

37. Fiers W, Beyaert R, Declercq W and Vandenabeele P: More than one way to die: apoptosis, necrosis and reactive oxygen damage. Oncogene 18: 7719-7730, 1999.

38. Simon HU, Haj-Yehia A and Levi-Schaffer F: Role of reactive oxygen species (ROS) in apoptosis induction. Apoptosis 5: 415-418, 2000

39. Panossian A, Hambardzumyan M, Hovhanissyan A and Wikman G: The adaptogens rhodiola and schisandra modify the response to immobilization stress in rabbits by suppressing the increase of phosphorylated stress-activated protein kinase, nitric oxide and cortisol. Drug Target Insights 2: 39-54, 2007. 